\title{
Proteinase 3-antineutrophil cytoplasmic antibody positive necrotizing vasculitis induced by ciprofloxacin
}

\author{
Khalid Ahmed $^{1 *}$, Syeda Atia Qudsia ${ }^{2}$, Syed Hani Abidi ${ }^{1}$, Rabia Malik ${ }^{1}$, \\ Muhammad Awais ${ }^{3}$, Abdul Rehman ${ }^{1}$
}

\begin{abstract}
${ }^{1}$ Department of Biological and
Biomedical Sciences, Aga Khan

University, Karachi, Pakistan

${ }^{2}$ Department of Medicine,

Liaquat National Medical

College and Hospital, Stadium

Road, Karachi, Pakistan

${ }^{3}$ Department of Radiology, Aga

Khan University Hospital,

Stadium Road, Karachi,

Pakistan
\end{abstract}

Received: 26 February 2016

Accepted: 04 April 2016

\section{*Correspondence to: \\ Dr. Khalid Ahmed, \\ Email: khalidmd.ahmed \\ @ gmail.com}

Copyright: (c) the author(s), publisher and licensee Medip Academy. This is an openaccess article distributed under the terms of the Creative Commons Attribution NonCommercial License, which permits unrestricted noncommercial use, distribution, and reproduction in any medium, provided the original work is properly cited.

\begin{abstract}
Granulomatosis with polyangiitis (Wegener's), microscopic polyangitis, and eosinophilic granulomatosis with polyangiitis are commonly grouped together as antineutrophil cytoplasmic antibody (ANCA)-positive vasculitides. Many drugs and infections can induce serologic positivity for ANCA, while a few can precipitate overt ANCA-positive vasculitis. Although fluoroquinolones have been reported to cause ANCA-negative leukocytoclastic vasculitis (LCV), fluoroquinolones are not known to induce proteinase 3-ANCA (PR3-ANCA)positive vasculitis. Here, we present the case of a middle-aged man who developed severe headache, purpura on legs and numbness in hands and feet after taking ofloxacin for 5 days. Subsequently, he was diagnosed with ANCAnegative LCV and treated with steroids and immunosuppressants. Thirteen years later, he inadvertently received intravenous ciprofloxacin and developed severe headache and epistaxis. Serologic testing at that time revealed elevated titers of PR3-ANCA. Biopsy of nasal septum revealed a mixed mononuclear and polymorphonuclear infiltrate without evidence of granuloma formation. He was treated with steroids and immunosuppressive therapy. Over the next several years, he remained stable with residual hearing loss and nasal septal deformity. This case provides the first evidence for a PR3-ANCA-positive necrotizing vasculitis induced by ciprofloxacin.
\end{abstract}

Keywords: Granulomatosis with polyangiitis, ANCA, Associated vasculitis, Cutaneous, LCV, Wegener's granulomatosis

\section{INTRODUCTION}

Granulomatosis with polyangiitis (Wegener's), microscopic polyangitis, and eosinophilic granulomatosis with polyangiitis are commonly grouped together as antineutrophil cytoplasmic antibody (ANCA)-positive vasculitides. Many drugs and infections can induce serologic positivity for ANCA, while a few can precipitate overt ANCA-positive vasculitis. ${ }^{1}$ Although ciprofloxacin can cause ANCA-negative leukocytoclastic vasculitis (LCV), fluoroquinolones are not known to induce proteinase 3-ANCA (PR3-ANCA)-positive vasculitis. Here, we present the case of a middle-aged man who developed PR3-ANCA-positive vasculitis after receiving ciprofloxacin. ${ }^{2}$

\section{CASE REPORT}

In 1995, a 32-year-old man presented with a 1-day history of severe headache and rash on his legs. He had completed a 5-day course of ofloxacin $(400 \mathrm{mg} \times 2 / \mathrm{d}) 1$ day prior to presentation for an upper respiratory tract 
infection. He reported numbness and loss of sensation in both hands and feet. On examination, he was febrile (temperature of $38.3^{\circ} \mathrm{C} ; 101^{\circ} \mathrm{F}$ ), but, his ENT examination was unremarkable. Palpable purpuras were noted over his left leg along with reduced sensation to pin-prick over the plantar surface of left foot. Laboratory investigations were notable for neutrophilic leukocytosis, positive anti-nuclear antibody (1:160), negative rheumatoid factor and normal ANCA levels. Punch biopsy of a skin lesion over the left medial malleolus revealed histopathologic features of LCV. Patient was prescribed an oral course of prednisolone $(1 \mathrm{mg} / \mathrm{kg})$ along with cyclophosphamide (pulse therapy) to which he responded well.

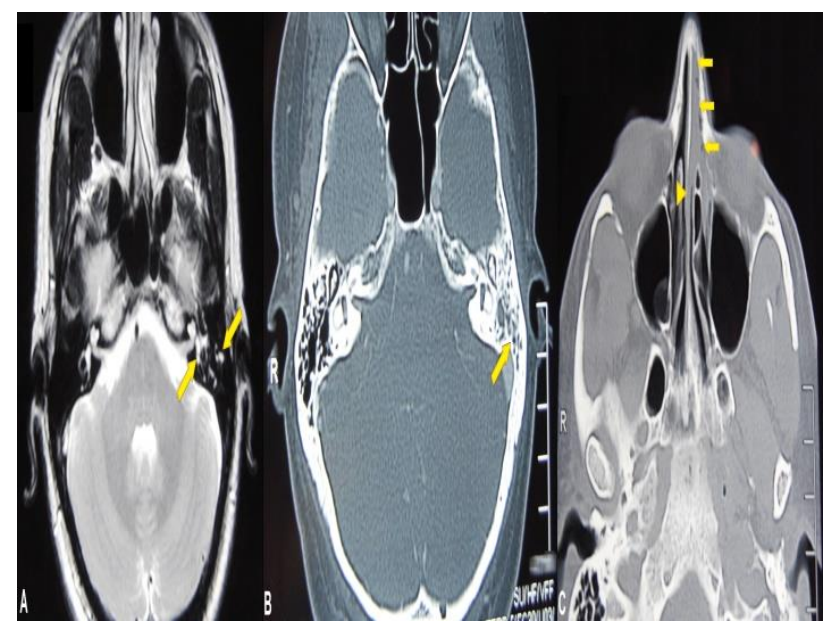

Figure 1: (A) Axial view of T2-weighted magnetic resonance image demonstrating the presence of fluid within left middle ear and mastoid air cells (arrows); (B, C) axial view of computed tomography showing; (B) opacification of mastoid air cells (arrow) and; (C) a soft-tissue density (arrows) within the nasal cavity causing erosion of nasal septum (arrowhead).

Thirteen years later, patient presented to the emergency department with blood-stained stools and was diagnosed with dysentery. Inadvertently, he received a single dose of intravenous ciprofloxacin $(400 \mathrm{mg})$ at this time. The following day he experienced severe headache, one episode of epistaxis and generalized weakness. There were no cutaneous eruptions or numbness of hands and feet at that time. He was evaluated in the otolaryngology clinic and prescribed analgesics and decongestants. However, his condition worsened further. MRI brain was performed, which showed fluid within mastoid air cells and middle ear [Figure 1(A)] along with a soft-tissue density within nasal cavity. CT paranasal sinuses was also ordered, which showed an infiltrative, soft-tissue density within the nasal cavity along with erosion of nasal septum [Figure 1(B) and 1(C)]. Laboratory investigations at this time were notable for neutrophilic leukocytosis, elevated liver enzymes, normal serum creatinine and positive ANCA (classic immunofluorescence pattern) directed against proteinase3. A nasal septal biopsy showed an admixture of mononuclear and polymorphonuclear infiltrate without any evidence of granuloma formation. Patient was treated with intravenous methylprednisolone $(1 \mathrm{~g} / \mathrm{d} \times 3)$ followed by an oral taper of prednisolone and immunosuppressive therapy. Over the next several years, patient remained stable with residual left-sided conductive hearing loss and nasal septal deformity.

\section{DISCUSSION}

In a comprehensive review of drug-induced rheumatologic disorders published, Bukhari identified four groups of drugs that commonly induce vasculitic disorders; these groups included anti-thyroid drugs, antitumor necrosis factor (TNF) medications, levamisoleadulterated cocaine and new small molecules. ${ }^{1}$ The spectrum of drug-induced vasculitis ranges from ANCAnegative vasculitis to ANCA-positive LCV to necrotizing neutrophilic dermatosis with positive antiphospholipid antibodies. $^{3-5}$ Drug-induced ANCA-positive vasculitis is often due to antibodies directed against myeloperoxidase, cathepsin G, lactoferrin or bacterial permeability increasing protein. ${ }^{6}$

Drug-induced necrotizing vasculitis due to PR3-ANCA has been rarely reported. Only a few drugs (propylthiouracil, methimazole, hydralazine, minocycline and levamisole-adulterated cocaine) have been previously reported to cause this severe vasculitic disorder. ${ }^{7-9}$ Fluoroquinolones are a generally well-tolerated class of drugs. In the literature, only a few cases have been reported hitherto regarding the development of fluroquinolone-induced vasculitis ${ }^{6}$ Storsley and Geldenhuys reported a rare case of ANCA-negative LCV induced by ciprofloxacin. ${ }^{10}$ To the best of our knowledge, the present case provides the first evidence for a PR3ANCA-positive necrotizing vasculitis induced by ciprofloxacin.

The strength of causal relationship of a drug with a probable adverse reaction is often judged by the Naranjo adverse drug reaction (ADR) probability scale. Based on responses to 10 items, a score is calculated regarding the likelihood of an ADR. Keeping the current case in perspective, a score of 8 can be calculated, which is consistent with a probable ADR. This score, when read in conjunction with previous reports of drug-induced vasculitis, provide strong evidence for a ciprofloxacininduced PR3-ANCA-positive vasculitic disorder.

\section{CONCLUSION}

Although rare, ciprofloxacin may induce a proteinase 3antineutrophil cytoplasmic antibody (PR3-ANCA)positive necrotizing vasculitis. Based on the evidence provided in this case, it would be prudent to avoid fluoroquinolones (whenever possible) in patients with a history of antineutrophil cytoplasmic antibody (ANCA)positive vasculitis. 
Funding: No funding sources

Conflict of interest: None declared

Ethical approval: The study was approved by the Institutional Ethics Committee

\section{REFERENCES}

1. Bukhari M. Drug-induced rheumatic diseases: a review of published case reports from the last two years. Curr Opin Rheumatol. 2012;24:182-6.

2. Reañto M, Vives R, Rodriguez J, Daroca P, Canto G, Fernandez J. Ciprofloxacin-induced vasculitis. Allergy. 1997;52:599-600.

3. Fujikawa K, Kawakami A, Hayashi T, Iwamoto N, Kawashiri S, Aramaki T, et al. Cutaneous vasculitis induced by TNF inhibitors: a report of three cases. Mod Rheumatol. 2010;20:86-9.

4. Hirohama D, Hoshino J, Hasegawa E, Yamanouchi M, Hayami N, Suwabe T, et al. Development of myeloperoxidase-antineutrophil cytoplasmic antibody-associated renal vasculitis in a patient receiving treatment with anti-tumor necrosis factor- $\alpha$. Mod Rheumatol. 2010;20:602-5.

5. Darne' S, Natarajan S, Blasdale C. Do antineutrophil cytoplasmic antibodies (ANCA) play a key role in neutrophilic dermatoses? A case of propylthiouracilinduced neutrophilic dermatosis with positive perinuclear ANCA. Clin Exp Dermatol. 2010;35:406-8.

6. Csernok E, Lamprecht P, Gross WL. Clinical and immunological features of drug-induced and infection-induced proteinase 3-antineutrophil cytoplasmic antibodies and myeloperoxidaseantineutrophil cytoplasmic antibodies and vasculitis. Curr Opin Rheumatol. 2010;22:43-8.

7. Nikolic BB, Nikolic MM, Andrejevic S, Zoric S, Bukilica M. Antineutrophil cytoplasmic antibody (ANCA)-associated autoimmune diseases induced by antithyroid drugs: comparison with idiopathic ANCA vasculitides. Arthritis Res Ther. 2005;7:R1072-81.

8. Agarwal G, Sultan G, Werner SL, Hura C. Hydralazine induces myeloperoxidase and proteinase 3 anti-neutrophil cytoplasmic antibody vasculitis and leads to pulmonary renal syndrome. Case Rep Nephrol. 2014;2014:868590.

9. Carlson AQ, Tuot DS, Jen KY, Butcher B, Graf J, Sam R, et al. Pauci-immune glomerulonephritis in individuals with disease associated with levamisoleadulterated cocaine: a series of 4 cases. Medicine. 2014;93:290-97.

10. Storsley L, Geldenhuys L. Ciprofloxacin-induced ANCA-negative cutaneous and renal vasculitisresolution with drug withdrawal. Nephrology Dialysis Transplantation. 2007;22:660-1.

Cite this article as: Ahmed K, Qudsia SA, Abidi SH, Malik R, Awais M, Rehman A. Proteinase 3antineutrophil cytoplasmic antibody positive necrotizing vasculitis induced by ciprofloxacin. Int J Basic Clin Pharmacol 2016;5:1145-7. 